\title{
Notes on specimens of the Glossy Mangrove Snake (Gerarda prevostiana) in the collection of the Zoological Survey of India, Kolkata along with past records from India
}

\author{
Probhat Bag*, Sujoy Raha and Kaushik Deuti \\ Zoological Survey of India, FPS Building, \\ $27 \mathrm{JL}$ Nehru Road, Kolkata - 700016, West Bengal, India
}

\section{Abstract}

Based on six specimens of the Glossy Mangrove Snake (Gerarda prevostiana) present in the collection of the Zoological Survey of India, Kolkata the species is redescribed with scalation and morphometric measurements and compared with data of six other specimens lodged in other museums.

Keywords: Infra-labial, Loreals, Post-ocular, Pre-ocular, Sub-caudals, Supra-labial

Gerarda prevostiana was described as Coluber (Homalopsis) prevostianus Eydoux and Gervais (1837) based on a specimen (currently untraced) from "Manile" (=Manila, $14^{\circ} 37^{\prime} \mathrm{N}, 120^{\circ} 58^{\prime} \mathrm{E}$, Luzon, Phillipines). The species is secretive and is known from isolated localities (mainly mangrove swamps of estuaries and tidal rivers) along both the coasts of India (Maharashtra, Kerala, Andhra Pradesh, Odisha, West Bengal and Andamans), coastal Sri Lanka, Bangladesh, Myanmar, Thailand, the Malay Peninsula and Sumatra and also the Phillipines (Tweedie, 1983; Murphy, 2007).

Its dietary habits, unique amongst snakes, include tearing out bite-sized pieces of recently molted softshelled crabs (Murphy, 2007). Small fishes and shrimps are also eaten (Das, 2002). They appear to co-occupy mounds of mud lobsters, Thalssinia anomala and $T$. Gracilis (Murphy, 2007) that also form part of their diet. However, there are few published records from India. One reason for the absence of records and the apparent rarity of this species within its overall distribution range may be due to its difficult to sample mangrove habitats (Das et al., 2013).
We examined all the specimens of this species present in the collection of the Reptilia Section at Zoological Survey of India, Kolkata and based on that provide here a description of the species along with its morphological details and also mention about past records from India.

Description based on specimens in ZSI, Kolkata (Registration Numbers: 13659, 22653, 23179, 24212, 25351 and 26145).

\section{Description}

Body is cylindrical with smooth scales. Head is slightly distinct from neck. Eyes are small situated high on the head with vertical pupil. Frontal scale much broader than supra-oculars. Loreal scale not in contact with the inter-nasal; a single inter-nasal separates the nasals. Seven supra-labials present, the eighth when present is very small, the fourth supra-labial touches the eye. Eight infralabials present, the ninth when present is very small, the fourth infra-labial contacts the first chin shield, which is much larger than the posterior chin shield. Anal divided. Tail short and terminating in a sharp tip.

\section{* Author for correspondence}


PLATE 1

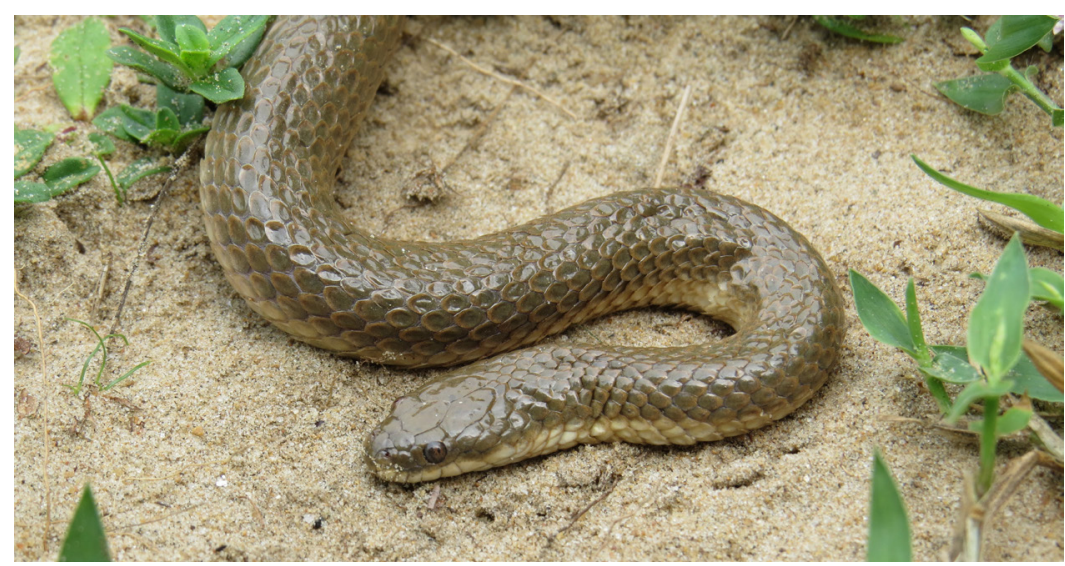

Figure 1. Gerarda prevostiana.

\section{Scalation}

The scalation of the ZSI specimens are given below in left/right order as follows:

\begin{tabular}{|c|c|c|c|c|c|c|}
\hline $\begin{array}{l}\text { Registration } \\
\text { Number }\end{array}$ & ZSI 13659 & ZSI 22653 & ZSI 23179 & ZSI 24212 & ZSI 25351 & ZSI 26145 \\
\hline Locality & $\begin{array}{l}\text { Howrah } \\
\text { near Kol- } \\
\text { kata }\end{array}$ & $\begin{array}{l}\text { Digha beach, East } \\
\text { Midnapurdistrict, West } \\
\text { Bengal }\end{array}$ & $\begin{array}{l}\text { Uppetera, Salt } \\
\text { Canal, Kakinada, } \\
\text { Andhra Pradesh }\end{array}$ & $\begin{array}{l}\text { Kakdwip, South } \\
24 \text { Parganas dis- } \\
\text { trict, West Bengal }\end{array}$ & $\begin{array}{l}\text { Cheepoo, } \\
\text { North Anda- } \\
\text { man }\end{array}$ & $\begin{array}{l}\text { Bichitrapur man- } \\
\text { groves, Balasore } \\
\text { district, Odisha }\end{array}$ \\
\hline Date of Collection & & 04.ix.1964 & 22.xi.1964 & 07.xi.1980 & 29.vi.2011 & 24.ix.2014 \\
\hline Name of Collector & Murray & A.K. Dutta & N.V. Subba Rao & N.C. Banerjee & $\begin{array}{l}\text { Sameer } \\
\text { Ghodke }\end{array}$ & Kaushik Deuti \\
\hline $\begin{array}{l}\text { Number of mid- } \\
\text { body scales }\end{array}$ & 17 & 17 & 17 & 17 & 17 & 17 \\
\hline $\begin{array}{l}\text { Number of ventral } \\
\text { scales }\end{array}$ & 152 & 151 & 157 & 150 & 150 & 148 \\
\hline $\begin{array}{l}\text { Number of } \\
\text { sub-caudal scales }\end{array}$ & 35 & 34 & 34 & 31 & 32 & 33 \\
\hline Loreals & $1 / 1$ & $1 / 1$ & $1 / 1$ & $1 / 1$ & $1 / 1$ & $1 / 1$ \\
\hline Pre-oculars & $1 / 1$ & $1 / 1$ & $1 / 1$ & $1 / 1$ & $1 / 1$ & $1 / 1$ \\
\hline Post-oculars & $2 / 2$ & $2 / 2$ & $2 / 2$ & $2 / 2$ & $2 / 2$ & $2 / 2$ \\
\hline Anterior loreals & $1 / 1$ & $1 / 1$ & $1 / 1$ & $1 / 1$ & $1 / 1$ & $1 / 1$ \\
\hline Posterior loreals & $2 / 2$ & $2 / 2$ & $3 / 3$ & $2 / 2$ & $2 / 2$ & $2 / 2$ \\
\hline Supra-labials & $8 / 8$ & $8 / 8$ & $7 / 8$ & $8 / 8$ & $7 / 7$ & $8 / 8$ \\
\hline Infra-labials & $9 / 9$ & $9 / 9$ & $8 / 8$ & $9 / 9$ & $8 / 8$ & $9 / 9$ \\
\hline
\end{tabular}

\section{Colour}

Dorsum unpatterned brown in four of our specimens preserved in alcohol, only specimen numbers ZSI 24212 and ZSI 26145 are with grey dorsum. Ventrum creamish.

\section{Measurements}

Measurements of specimens in Zoological Survey of India are given below (all measurements in millimetres): 


\begin{tabular}{|l|c|c|c|c|c|c|}
\hline $\begin{array}{l}\text { Registration } \\
\text { Number }\end{array}$ & ZSI 13659 & ZSI 22653 & ZSI 23179 & ZSI 24212 & ZSI 25351 & ZSI 26145 \\
\hline Snout-vent length & 445 & 466 & 467 & 332 & 380 & 365 \\
\hline Tail length & 66 & 66 & 84 & 54 & 54 & 64 \\
\hline Head length & 10.31 & 11.98 & 12.04 & 9.40 & 9.33 & 9.81 \\
\hline Head width & 8.54 & 10.92 & 12.02 & 8.03 & 8.65 & 8.99 \\
\hline Snout length & 4.31 & 6.38 & 6.20 & 4.67 & 4.78 & 5.32 \\
\hline Eye-nostril length & 2.55 & 3.58 & 3.53 & 2.65 & 2.52 & 2.73 \\
\hline Nostril-snout tip length & 1.62 & 2.84 & 2.39 & 1.40 & 1.98 & 1.97 \\
\hline Eye diameter & 1.99 & 2.48 & 2.56 & 1.84 & 1.53 & 2.05 \\
\hline Inter-narial length & 2.06 & 2.76 & 3.13 & 2.13 & 2.31 & 2.08 \\
\hline Inter-orbital length & 4.63 & 6.35 & 6.10 & 4.77 & 5.02 & 5.20 \\
\hline
\end{tabular}

Details of specimens with past records from India (all measurements in millimetres):

\begin{tabular}{|c|c|c|c|c|c|c|}
\hline $\begin{array}{l}\text { Registration } \\
\text { Number }\end{array}$ & & & & & & \\
\hline Locality & $\begin{array}{l}\text { Bandaora, } \\
\text { Bombay coast, } \\
\text { Maharashtra }\end{array}$ & $\begin{array}{l}\text { Cannanore } \\
\text { (now Kannur), } \\
\text { Kerala }\end{array}$ & $\begin{array}{l}\text { Backbay, Bom- } \\
\text { bay, Maharashtra }\end{array}$ & $\begin{array}{l}\text { Versova, Salsette, } \\
\text { Maharashtra }\end{array}$ & $\begin{array}{l}\text { Chaupati } \\
\text { Sands, Bom- } \\
\text { bay }\end{array}$ & $\begin{array}{l}\text { Sion, Bom- } \\
\text { bay }\end{array}$ \\
\hline Date of Collection & & 08.xi.1903 & 1923 & 1923 & 1923 & 1923 \\
\hline Name of Collector & H.M. Phipson & $\begin{array}{l}\text { Ferdinand } \\
\text { Wall }\end{array}$ & J. Mann & & J.P. Mullan & J.P. Mullan \\
\hline Lodged in Museum & $\begin{array}{l}\text { British Museum, } \\
\text { London }\end{array}$ & & \multicolumn{4}{|c|}{ Bombay Natural History Society, Mumbai } \\
\hline Reference & $\begin{array}{l}\text { Boulenger } \\
(1896)\end{array}$ & Wall (1905) & \multicolumn{4}{|l|}{ Prater (1924) } \\
\hline Number of mid-body scales & NA & NA & NA & NA & NA & NA \\
\hline Number of ventral scales & 154 & 151 & \multicolumn{4}{|l|}{$146-158$} \\
\hline Number of sub-caudal scales & 33 & 31 & \multicolumn{4}{|l|}{$31-34$} \\
\hline Snout-vent length & & 498 & & & & \\
\hline Tail length & & 63.5 & & & & \\
\hline
\end{tabular}

\section{Acknowledgements}

The authors are grateful to Director, ZSI and Officer-In-charge, FPS Building, ZSI, Kolkata for laboratory facilities.

\section{References}

Boulenger, G.A. 1896. Catalogue of the snakes in the British Museum (Natural History). Taylor and Francis, London. Volume 3. 721 pp.

Das, I. 2002. Snakes and other reptiles of India. New Holland publishers, United Kingdom. 144 pp.

Das, I., Breuer, H. and Shonleben, S. 2013. Gerarda prevostiana (Eydoux and Gervais, 1837) (Squamata: Serpentes: Homalopsidae), a new snake from Borneo. Asian Herp Research., 4(1): 76-78.

Murphy, J.C. 2007. Homalopsid snakes: Evolution in the mud. Krieger Publishing Company, Florida. 249 pp.

Prater, S.H. 1924. The snakes of Bombay Island and Salsette. J. Bombay Nat Hist Soc., 30: 151-176.

Tweedie, M.W.F. 1983. The snakes of Malaya. $3^{\text {rd }}$ edition. Singapore National printers. $167 \mathrm{pp}$.

Wall, F. 1905. Notes on snakes collected in Cannanore from $5^{\text {th }}$ November, 1903 to $5^{\text {th }}$ August, 1904. J. Bombay Nat Hist Soc., 16: 292317.

Whitaker, R and Captain, A. 2004. Snakes of India - the field guide. Drace books, Chennai, India. 253 pp. 